\title{
Power and Modulation Assignment via Perron-root Optimization for Interference Limited Systems
}

\author{
Eduardo Castañeda, Ramiro Samano-Robles, and Atílio Gameiro, \\ Instituto de Telecomunicações, Campus Universitário, Aveiro, 3810-193, Portugal. \\ \{ecastaneda, ramiro\}@av.it.pt, amg@ua.pt
}

\begin{abstract}
The maximization of the total sum rate depends on the proper power and modulation assignment. The feasibility of such resource assignment is susceptible to the set of links that are attempted to be scheduled. In this paper we address the problem of maximizing the sum rate while guaranteeing resource assignment feasibility in interference limited wireless systems. Unlike the current literature, the allowed signal-tointerference-plus-noise ratio is constrained to take values from a finite set associated with a finite number of available modulations. Therefore, we present suboptimal but efficient algorithms to solve the joint user selection and resource (power and modulation) assignment, which is a combinatorial problem. The feasibility of the resource assignment is verified by a criterion based on the Perron-Frobenius theory whilst the optimization of the resource allocation is achieved either by user-removal like techniques or by a novel criterion derived from the Perron-Frobenius theory. We provide numerical results to confirm the efficiency of our resource allocation algorithms compared to the optimal resource allocation.
\end{abstract}

\section{INTRODUCTION}

In the literature of wireless communications, an interference limited wireless system is described as a set of transmitterreceiver pairs operating simultaneously in a shared medium. Recent works on resource allocation optimization for interference limited systems show how network utility maximization [1]-[3], individual rate maximization [4], [5], and their mathematical characterization [4]-[8] are intimately related to power control. The total rate maximization and individual rate maximization problems are explicitly constrained by a minimum adequate quality of service (QoS) that in practice is measured by the signal-to-interference-plus-noise ratio (SINR). All data rates are coupled by the individual power of each link, making the SINR a global function of all transmit powers.

In several papers (e.g., [1]-[4]) the optimization of the resource allocation problem has been tackled by means of the Perron-Frobenius theory of nonnegative matrices [9], [10] assuming that all QoS requirements are feasible. The power and rate allocation problem can be reformulated as an Perronroot optimization problem [4], [6] whose solution exists as long as the SINR requirements of all links are achievable under a set of power constraints. Nevertheless, the required SINRs are not always feasible and therefore algorithms for resource assignment may not converge when the QoS requirements of all links cannot be met simultaneously. The proper

The work presented in this paper was supported by the Portuguese COPWIN (PTDC/EEI-TEL/1417/2012) and CROWN (PTDC/EEATEL/115828/2009) projects. selection of the subset of links whose QoS requirements are jointly achievable requires the verification of some feasibility conditions and the temporary dropping of links that violate them. This process is known as user-removal [7], [11], and its goal is to define the subset of links that maximizes a given metric assuming fixed SINR targets that can be fully satisfied. Finding this optimum subset is a NP-complete problem [7].

The works related to the resource allocation optimization based on the Perron-Frobenius theory and the ones of link removal have different objectives. The former assumes that the solution of the resource allocation problem exists, consequently, only the optimization of the rate and power allocation is sought. The latter is concerned about the subset of links for which a given resource allocation exists and such allocation can be achieved by simple assignment schemes. In this work we combine both aspects of the problem: feasibility is verified by a criterion based on the Perron-Frobenius theory while sum rate maximization is achieved by optimizing the powers and modulations either by user-removal like techniques or by a novel criterion derived from the Perron-Frobenius theory. Furthermore, instead of assuming that the SINR targets take values from a continuous range (e.g., [1]-[7]), a finite set of modulation and coding schemes (MCS) is considered, which to the best of our knowledge, has not been addressed by means of the Perron-Frobenius theory for interference coupled systems. The assignment of modulations (or rates) and powers becomes a combinatorial problem that grows exponentially with the number of links and depends on the cardinality of the set of targets. Therefore, we propose suboptimal but rather efficient algorithms that define a subset of links that achieve a feasible power and rate allocation and a sum rate performance close to the optimum one.

Some notational conventions are as follows: matrices and column vectors are set in boldface. $(\cdot)^{T},|\cdot|$ denote transpose and set cardinality respectively. A matrix $\mathbf{A}$ is nonnegative if $a_{m, n} \geq 0, \forall m, n$ and write $\mathbf{A} \geq \mathbf{0}$. The term $\rho(\mathbf{A})$ denotes the Perron-Frobenius root (PF-root) which equals the largest modulus eigenvalue of the matrix $\mathbf{A}$ [9], [10]. $\mathbf{I}$ is the identity matrix of compatible size. $\operatorname{diag}(\mathbf{x})$ denotes a diagonal matrix whose main diagonal is $\mathbf{x} . \mathbf{A}^{[i]}$ is the $i^{t h}$ principal submatrix of A whose $i^{\text {th }}$ row and column are removed. The same notation is applied for a vector whose $i^{\text {th }}$ element is removed. Let $\mathbf{y}$ be a vector, then $y_{i}=[\mathbf{y}]_{i}$ is the $i^{t h}$ element. For two vectors $\mathbf{x}$ and $\mathbf{y}, \mathbf{x} \geq \mathbf{y}$ is a componentwise inequality. The set $\mathbb{R}_{++}$ are strictly positive real numbers. 


\section{System Model}

We assume that the current channel instance is concurrently being employed by $K$ synchronized links. The receivers decode its corresponding data treating interference as white noise and multiuser detection is not employed. We adopt the matrix notation and the system model used in recent works [1], [6]. Given a power vector $\mathbf{p}$, the SINR experienced in the $k^{\text {th }}$ link is [6]:

$$
\operatorname{SINR}_{k}(\mathbf{p})=\frac{p_{k} G_{k k}}{\sum_{i \neq k}^{K} p_{i} G_{k i}+\sigma_{k}^{2}},
$$

where $G_{k i}$ is the power attenuation from the transmitter on link $i$ to the receiver on link $k$, taking into account propagation loss, fast and slow fading. $G_{k k}$ is the power loss for the intended transmission on link $k$. The term $\sigma_{k}^{2}$ represents the additive white Gaussian noise (AWGN) power for the $k^{\text {th }}$ receiver.

The resource allocation policies seek the simultaneous provisioning of individual QoS for multiple wireless links, which implies that each link achieves a SINR that can be maintained above a given threshold or target:

$$
S I N R_{k}(\mathbf{p}) \geq \gamma_{k} .
$$

The SINR target of the $k^{\text {th }}$ link is constrained to take values from a finite set of targets $\gamma_{k} \in \mathcal{M}_{k}=\left\{\gamma^{(1)}, \ldots, \gamma^{(M)}\right\}$ where $\gamma^{(m-1)}<\gamma^{(m)}$ and $M$ is the number of available modulations. A larger value of $\gamma_{k}$ implies that link $k$ attempts to maintain a more spectral efficient modulation scheme. Without loss of generality we assume the same $\mathcal{M}$ for all links, i.e., $\mathcal{M}_{k}=\mathcal{M}, \forall k$. The $k^{t h}$ link is associated with a modulation index $m_{k}$ that defines the position of its SINR target in the set $\mathcal{M}$ so that $\gamma_{k}=\gamma^{\left(m_{k}\right)}$. The set of targets is given by the available set of MCS supported in the systems, which in practice is defined by the user equipment capabilities and the wireless network technology. The vector of SINR targets is defined as $\gamma=\left(\gamma_{1}, \ldots, \gamma_{K}\right)^{T}$. The SINR targets will be summarized in a diagonal matrix $\boldsymbol{\Gamma}=\operatorname{diag}(\gamma)$.

The users' requirements in (2) can be described in a vector inequality of the form:

$$
\mathbf{p} \geq \Gamma \mathbf{V p}+\Gamma \mathbf{z}
$$

where $\mathbf{V}$ is a $K \times K$ nonnegative matrix whose entries are defined as $[\mathbf{V}]_{k i}=G_{k i} / G_{k k}$, if $k \neq i$ and $[\mathbf{V}]_{k i}=0$, if $k=i$. We assume that $\mathbf{V}$ is irreducible, which means that each link has at least one interferer [5]. The weighted noise vector is defined as $\mathbf{z}=\left(\sigma_{1}^{2} / G_{11}, \ldots, \sigma_{K}^{2} / G_{K K}\right)^{T}$. We consider individual power constraints summarized in $\overline{\mathbf{p}}$ so that $\bar{p}_{k}$ is the maximum available power for the $k^{t h}$ link. Let $\mathbf{B}_{k}$ be a $K \times K$ nonnegative irreducible matrix that absorbs the power constrains of the $k^{\text {th }}$ link as an additional source of interference, and it is defined as $[6, \S 5]$ :

$$
\mathbf{B}_{k}=\boldsymbol{\Gamma}\left(\mathbf{V}+\frac{1}{\bar{p}_{k}} \mathbf{z e}_{k}^{T}\right),
$$

where $\mathbf{e}_{k}$ is an indicator vector with all elements equal to zero and the $k^{t h}$ element equal to one.

\section{PRoblem Formulation}

From (2) and (3) it can be observed that the power vector $\mathbf{p}$ and the achievable SINR depend on the targets $\boldsymbol{\Gamma}$. From power control theory [5], [6], [8] and Perron Frobenius theory [9], [10], it is known that for feasible targets in (2), the power allocation vector is given by:

$$
\mathbf{p}=[\mathbf{I}-\mathbf{\Gamma V}]^{-1} \mathbf{\Gamma z} .
$$

Feasibility of the SINR targets means that all elements in $\gamma$ can be jointly achieved by power control. Considering individual power constraints given by $\overline{\mathbf{p}}$, the solution of (5) must be within the the region of feasible powers defined as:

$$
\mathcal{P}^{I C}:=\left\{\mathbf{p} \in \mathbb{R}_{++}^{K}: \mathbf{p} \leq \overline{\mathbf{p}}\right\},
$$

where $K=|\mathcal{K}|$ is the cardinality of the subset of links that can be jointly supported $\mathcal{K} \subseteq \overline{\mathcal{K}}$, and $\overline{\mathcal{K}}$ is the set of all available links. We need to solve the following rate maximization problem over the set of feasible links $\mathcal{K}$ constrained in the joint continuous power and discrete target regions:

$$
\begin{array}{cl}
\text { maximize } & \sum_{k \in \mathcal{K}, \mathcal{K} \subseteq \overline{\mathcal{K}}} w_{\boldsymbol{\gamma}} R\left(S I N R_{k}(\mathbf{p})\right) \\
\text { subject to } & \gamma_{k} \in \mathcal{M}, \quad \forall k \in \mathcal{K} \\
& \mathbf{p} \in \mathcal{P}^{I C},
\end{array}
$$

where $R(\cdot)$ is the rate associated with a given SINR [12] and $w_{\gamma}$ is a priority weight associated with $\gamma$ and the constraints imposed to subset $\mathcal{K}$.

From the Perron-Frobenius theory [9], [10], for a nonnegative square matrix $\mathbf{A}$, its PF-root $\rho(\mathbf{A})$ and its associated right eigenvector $\mathbf{x}$ meet: $\mathbf{A x} \leq \mathbf{x}$, if and only if $\rho(\mathbf{A}) \leq 1$. In our context, this property means that the SINR targets $\gamma$ are jointly achievable if and only if the following necessary and sufficient condition for feasibility is met [6]:

$$
\max _{k \in \mathcal{K}} \rho\left(\mathbf{B}_{k}\right) \leq 1 .
$$

Fulfilling (8) implies that interference in the system can be mitigated by power control, i.e., the SINR targets are feasible and (2) holds with equality. Furthermore, the power vector $\mathbf{p}$ given by (5) equals the right eigenvector associated with $\rho\left(\mathbf{B}_{k}\right)$ for all $k \in \mathcal{K}[6]$. The feasible region of SINR targets can be characterized as follows:

$$
\mathcal{Q}^{I C}:=\left\{\gamma_{k}=\gamma^{\left(m_{k}\right)} \in \mathcal{M}, \forall k \in \mathcal{K}: \max _{k \in \mathcal{K}} \rho\left(\mathbf{B}_{k}\right) \leq 1\right\}
$$

The existence of a feasible power vector is guaranteed always that $\gamma \in \mathcal{Q}^{I C}$. If (8) is met there exists a $\mathbf{p}$ that fulfils the power constraints (6) and all SINR requirements. Therefore, problem (7) can be described as a PF-root optimization over the matrices $\mathbf{B}_{k}$ (4) and reformulated as:

$$
\begin{array}{ll}
\text { maximize } & \sum_{k \in \mathcal{K}, \mathcal{K} \subseteq \overline{\mathcal{K}}} w_{\boldsymbol{\gamma}} R\left([\gamma]_{k}\right) \\
\text { subject to } & \gamma \in \mathcal{Q}^{I C}
\end{array}
$$

Since the elements of $\gamma$ can only take values from a finite set, (10) is a combinatorial problem whose complexity depends on the size of $\mathcal{M}$ and the number of elements in $\overrightarrow{\mathcal{K}}$. 


\section{Resource Assignment Algorithms}

Finding a solution to (10) requires the optimization over the set of feasible links that can transmit simultaneously (user selection) and their respective feasible modulations (and their associated powers). An exhaustive search algorithm attempting to solve (10) would require to test all possible subsets of links and all combination of target vectors for each subset. The associated search space $\Omega_{\overline{\mathcal{K}}, M}$ has a size of $(M+1)^{|\overline{\mathcal{K}}|}-1$, where several configurations of links and target vectors are infeasible, i.e., $\gamma \notin \mathcal{Q}^{I C}$. As the computation of the optimum solution is prohibitive, we develop suboptimal algorithms whose solutions approximate to the optimal one.

In the algorithms we adopt the notation $\Xi(k, \mathcal{K}, \mathbf{V}, \mathbf{z}, \mathbf{p}, \gamma)$ to indicate a dropping event of the link $k$ and consequent actions follow: $\mathcal{K}=\mathcal{K}-\{k\}, \mathbf{V}=\mathbf{V}^{[k]}, \boldsymbol{\gamma}=\boldsymbol{\gamma}^{[k]}, \mathbf{z}=\mathbf{z}^{[k]}$, $\mathbf{p}=\mathbf{p}^{[k]}$, and $\gamma_{i}=\gamma^{\left(m_{i}=M\right)} \forall i \in \mathcal{K}$.

\section{A. Optimal Resource Allocation}

The optimal solution for problem (10) can be given by two different approaches depending on the constraints over the final subset of feasible links. On the one hand, if the size of $\mathcal{K}$ is not constrained, then the optimal solution $\gamma^{\star}$ is unique and the priority weights are simply $w_{\gamma}=1, \forall \gamma$. On the other hand, if the size of $\mathcal{K}$ is constrained to be maximum, then it may exist a set of target vectors with equal sum rates and equal maximum cardinality. Therefore, we consider two criteria to reach the optimum allocation: one, by making $\gamma^{\star}$ equal to any $\gamma$ with the maximum cardinality and sum rate, which implies $w_{\gamma}=1, \forall \gamma$. And two, by taking into account the rate distribution among the links in each $\mathcal{K}$, and $\gamma^{\star}$ is given by:

$$
\boldsymbol{\gamma}^{\star}=\arg \max _{\left\{\boldsymbol{\varrho}^{\{\mathcal{K}\}}\right\} \in \Omega_{\overline{\mathcal{K}}, M}} \sum_{k \in \mathcal{K}} w_{\boldsymbol{\varrho}^{\{\mathcal{K}\}}} R\left(\left[\boldsymbol{\varrho}^{\{\mathcal{K}\}}\right]_{k}\right),
$$

where $w_{\boldsymbol{\rho}^{\{\mathcal{K}\}}}$ is a fairness weight associated with the targets $\varrho^{\{\mathcal{K}\}}$ of the subset $\mathcal{K}$ and is given by the Jain fairness index [13] of the data rates defined by $\varrho^{\{\mathcal{K}\}}$. The optimum solution $\gamma^{\star}$ found by (11) maximizes both the product sum rate by fairness index and the final number of active links.

\section{B. Target relaxation for a non-fixed subset of links}

From the theory of irreducible matrices it is known that if $\mathbf{A}$ is an irreducible square matrix and $\mathbf{A}^{[k]}$ is a proper principal submatrix of $\mathbf{A}$, then $\rho\left(\mathbf{A}^{[k]}\right)<\rho(\mathbf{A})$ [9], [10]. This property is exploited in the context of user removal [7] to find the worst link that conditions the fulfillment of (8). The user removal techniques are used to select a subset of users that can satisfy their SINR requirements simultaneously. The link $k^{*}$ that must be dropped is the one that creates maximum interference without achieving its assigned target. Such a link has the maximum PF-root $\rho\left(\mathbf{B}_{k^{*}}\right)$ and imposes infeasibility to the subset $\mathcal{K}$. In our context, we identify the most infeasible component $k^{*}$ in the target vector $\gamma$ by finding the link $k^{*}$ with maximum PF-root over the set of matrices defined by (4). The SINR target of the link $k^{*}$ is relaxed so that the maximum PF-root for the next set of matrices (4) is minimized.
We set $w_{\gamma}=1, \forall \gamma$ since neither the cardinality of the final $\mathcal{K}$ nor the distribution of the achievable rates among the links in $\mathcal{K}$ are considered. The initial conditions of the targets are set to $\gamma_{k}=\gamma^{\left(m_{k}=M\right)}, \forall k \in \mathcal{K}$ which may be or not a feasible starting point and is the maximum available target defined by any given set $\mathcal{M}$. The feasibility of the targets is verified by (8), if $\gamma \in \mathcal{Q}^{I C}$ then the algorithm stops and all links in $\mathcal{K}$ transmit simultaneously with the powers defined by (5). If $\gamma$ is infeasible, a relaxation of the SINR targets is required and each iteration the algorithm modifies only one component $[\gamma]_{k^{*}}$, where the most infeasible link $k^{*}$ is found by [7]:

$$
k^{*}=\arg \max _{k \in \mathcal{K}}\left(\min _{i \in \mathcal{K}, i \neq k} \frac{1}{\rho\left(\mathbf{B}_{i}^{[k]}\right)}\right) .
$$

For the next iteration the link $k^{*}$ reduces its target index by one, $m_{k^{*}}=m_{k^{*}}-1$, and its new SINR target is set to $\gamma_{k^{*}}=\gamma^{\left(m_{k^{*}}\right)}$. In the case that link $k^{*}$ cannot reduce its minimum target, then it is classified as infeasible and dropped. By assigning any positive power allocation to this link will only create interference to the other links without achieving the minimum required SINR. The set of feasible links is reduced so that $\mathcal{K}=\mathcal{K}-\left\{k^{*}\right\}$, and without the useless link, the algorithm attempts to allocate the maximum available target for the remaining users, i.e., $m_{k}=M, \forall k \in \mathcal{K}$. The procedure to solve (10) is described in Algorithm 1.

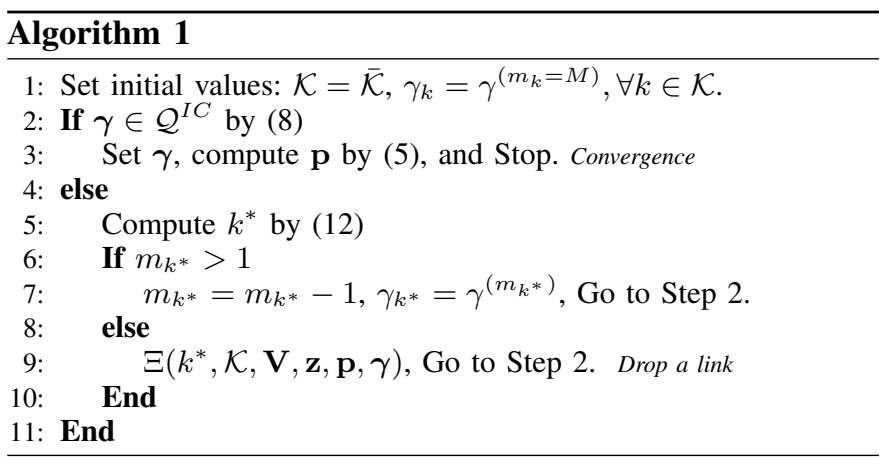

Algorithm 1 looks for the maximum SINR target vector closer to the boundary of $\mathcal{Q}^{I C}$. The target vector $\gamma$ found by this approach is not necessary the same size of the optimal one $\gamma^{\star}$. If $\left|\gamma^{\star}\right| \neq|\gamma|$ then the feasible regions (9) of both solutions are different. This algorithm is only suitable for centralized wireless networks as it requires a central network controller that has a knowledge of global network parameters to compute the PF-root. In this approach, $\left(|\mathcal{K}|^{2}-|\mathcal{K}|\right)$ PFroot computations are required each iteration $\left(\mathcal{O}\left(|\mathcal{K}|^{3}\right)\right.$ ) and the maximum number of iterations require to find $\mathcal{K}$ is upper bounded by $\left(\sum_{i=1}^{K}((M-1) i+1)\right)-1$.

\section{Target increment for a fixed subset of links}

In this approach the resource assignment starts with a feasible target vector and the objective is to increase its components as much as possible maintaining feasibility. Since $\mathcal{Q}^{I C}$ is downward comprehensive [6] the vector $\gamma$ found by Algorithm 1 defines a set of vectors which are inside the 
feasible region $\mathcal{Q}^{I C}$. However, if the initial conditions of $\gamma$ are set to the minimum attainable target, the unique explicit information about how far these initial conditions are from the boundary of $\mathcal{Q}^{I C}$ is given by (8). Therefore, the problem is how to tighten up the SINR requirements in order to solve (10) keeping fixed the initial feasible subset of links $\mathcal{K}$ ?

Proposition 1: Consider that $\gamma \in \mathcal{Q}^{I C}$. The element $\gamma_{k^{*}}$ that can be increased yielding the minimum increment of the left-hand side of (8) is the one whose $\rho\left(\mathbf{B}_{k^{*}}\right)$ is minimum.

Proof: Let $i$ be the element whose $\rho\left(\mathbf{B}_{i}\right)$ is maximum. According to [7, Thm. 9] the maximum achievable $\gamma \in \mathcal{Q}^{I C}$ is defined by $\bar{p}_{i}$ and the power $p_{i}$ associated with $\gamma_{i}$ is maximum, i.e., $i=\arg \min _{k} \bar{p}_{k}-p_{k}$ [1, Thm. 2]. Let $j=\arg \min _{k} \rho\left(\mathbf{B}_{k}\right)$, and $\gamma_{(k)} \in \mathcal{Q}^{I C}$ where $\gamma_{(k)}=\gamma+\delta \mathbf{e}_{k}$ is the target vector whose $k^{t h}$ term increases $\delta \in \mathbb{R}_{++}$. Since (8) identifies which is the tightest power constraint, i.e., which $\bar{p}_{k}$ is the hardest to meet, and an increment in $\gamma_{(k)}$ requires an increment in $p_{k}$, making $\gamma_{(j)}$ will increase the power $p_{j}$ associated with the less tight power constraint $\bar{p}_{j}$.

In this approach the minimum available target is initially assigned to all links and we set $w_{\gamma}=1, \forall \gamma$. The links that cannot meet this minimum SINR requirement are found by (12) and dropped. Once that the initial subset of feasible links $\mathcal{K}$ has been defined, it follows from Proposition 1 that the link $k^{*}$ candidate to increase its SINR requirements is given by:

$$
k^{*}=\arg \min _{k \in \mathcal{K}} \rho\left(\mathbf{B}_{k}\right) .
$$

An approximation to (13) that avoids the $|\mathcal{K}|$ eigenvalue computations is to define $k^{*}$ as the link with with less power consumption relative to its power constraint:

$$
k^{*}=\arg \max _{k \in \mathcal{K}}\left(\bar{p}_{k}-p_{k}\right) / \bar{p}_{k} .
$$

Notice that this simplification applies since $\gamma$ and $\mathbf{p}$ are assumed feasible. In the case of Algorithm 1 the vector of powers in the feasible power region (6) that satisfies all SINR requirements exists only if (8) is met and it cannot be used directly to make previous decision. The tightening of the targets is performed stepwise until (8) cannot be fulfilled, which implies $|\mathcal{K}|$ PF-root computations $\left(\mathcal{O}\left(|\mathcal{K}|^{3}\right)\right)$ each iteration as described in Algorithm 2.

\section{Numerical ExAmple}

To evaluate the performance of the algorithms we use a distributed antenna system (DAS). The deployment consists of $N=4$ distributed remote antenna units (RAUs), one RAU at the center of the cell and 3 RAUs uniformly deployed at a distance from the cell center of $\frac{2}{3}$ the cell radius. We consider that the RAUs are coordinated only to control their transmit powers and no signal processing is used (e.g., beamforming). The channels are modeled as Rayleigh fading and affected by a path-loss component and a shadowing fading component modeled as a log-normal distributed variable with standard deviation $s_{\sigma}$. There are $U$ users uniformly deployed in the cell. The initial set of links $\overline{\mathcal{K}}$ is defined according to the algorithm for RAU-user matching presented in [14]. After all

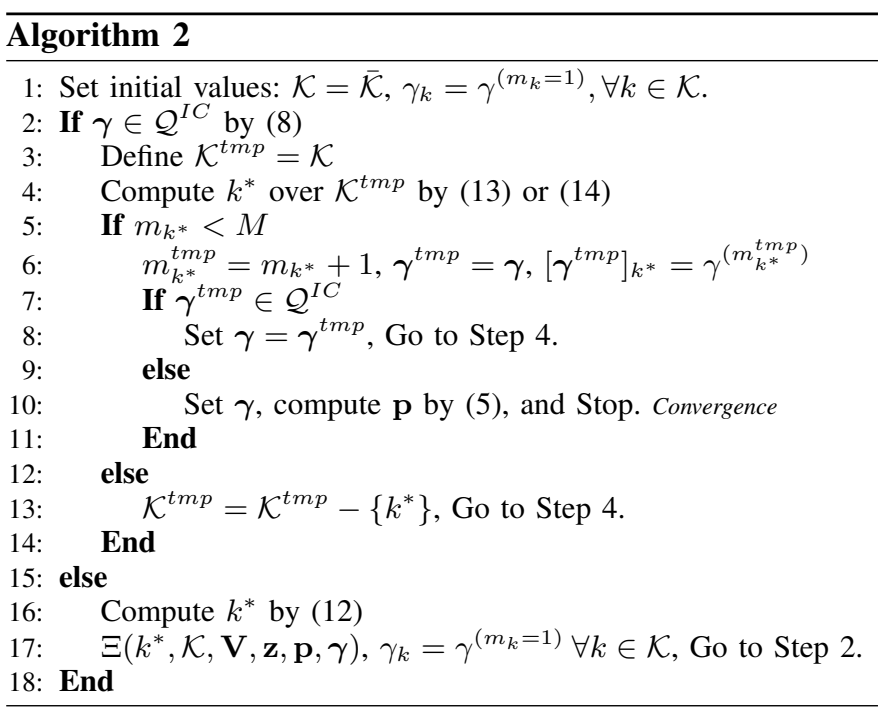

RAU-user links have been established, Algorithms 1 and 2 are performed. The set of available SINR targets or thresholds is given by $\mathcal{M}=\{-3.2,1.8,5.0,7.2,11.2,14.8,19.0,22.8\}$ in $\mathrm{dB}$ [12]. Results are generated by averaging 10000 channel instances for each value of $U$ and the simulation parameters are listed in Table I.

TABLE I: Simulation Parameters

\begin{tabular}{lc||lc}
\hline Parameters & Values & Parameters & Values \\
\hline Cell radius & $900 \mathrm{~m}$ & User deployment & uniform \\
Channel BW & $20 \mathrm{MHz}$ & Carrier freq. & $2.5 \mathrm{GHz}$ \\
$\bar{p}$ per RAU & $43 \mathrm{dBm}$ & Thermal noise $N_{0}$ & $-174 \mathrm{dBm} / \mathrm{Hz}$ \\
UE noise figure & $7 \mathrm{~dB}$ & MCS $(M)$ & $8[12]$ \\
Path loss model & UMi-LoS $[15]$ & Shadow fading & $s_{\sigma}=3$ \\
\hline
\end{tabular}

In Fig. 1 the performance in terms of sum rate versus the user diversity is presented. The variation in the total number of users $U$ is used to study user diversity, i.e., as $U \rightarrow \infty$ the channel conditions of the users attached to the RAUs are improved. Algorithm 1 solves (10) regardless $\mathcal{K}$ whilst Algorithm 2 also maximizes the cardinality of $\mathcal{K}$. The solutions found by both approaches are expected to present large differences in terms of sum rate for values of $U \approx N$ since the initial set $\overline{\mathcal{K}}$ has links whose channel conditions may be unfavorable. Nevertheless, for the case $U=N$ both algorithms have a rate gap less than $10 \%$ compared to the optimal allocation. For $U \gg N$ both approaches converge to similar values of sum rate due to user diversity, which also implies that more links can achieve the maximum modulation scheme. The sum rate gap between Algorithm 2 via (14) and via (13) is negligible (less than $2 \%$ ) which is a substantial gain in term of complexity when (14) is used.

The Fig. 2 presents the cumulative distribution function of the sum rate for $U=8$. Two particular case are shown: (a) for a fixed sum rate target of $15 \mathrm{bps}$, the gap between the proposed algorithms and the optimal allocation is less than $10 \%$ which is an acceptable approximation considering all available combinations of links subsets and targets $\left(\left|\Omega_{|\overline{\mathcal{K}}|=4, M=8}\right|=6560\right)$. 
(b) for a required probability $\operatorname{Pr}=0.3$, the sum rate gap is less than $10 \%$ for both proposed algorithms when compared to the optimum allocation.

The outage probability has been used as a metric to assess the performance of different power control algorithms and it can be defined as the ratio between the number of dropped links, to the total number of links [7]. The performance in terms of outage probability is presented in Fig. 3. For a fixed outage probability target of $10^{-3}$ it can be observed that the approach used by Algorithm 2 exploits more efficiently user diversity compared to the approach of Algorithm 1. Algorithm 2 can be used to minimize outage since it requires $U>8$ to make $|\mathcal{K}|=N$ and it achieves a performance similar to the optimal allocation for fixed subset $\mathcal{K}$. In contrast, Algorithm 1 requires $U>28$ to achieve zero outage which may be a tough condition to fulfil. Notice that the optimum allocation for a non-fixed $\mathcal{K}$ requires $U \gg N$ to achieve zero outage. The sum rate deficiency of Algorithm 2 compared to Algorithm 1 is compensated in terms of fairness since $|\mathcal{K}|$ is also maximized.

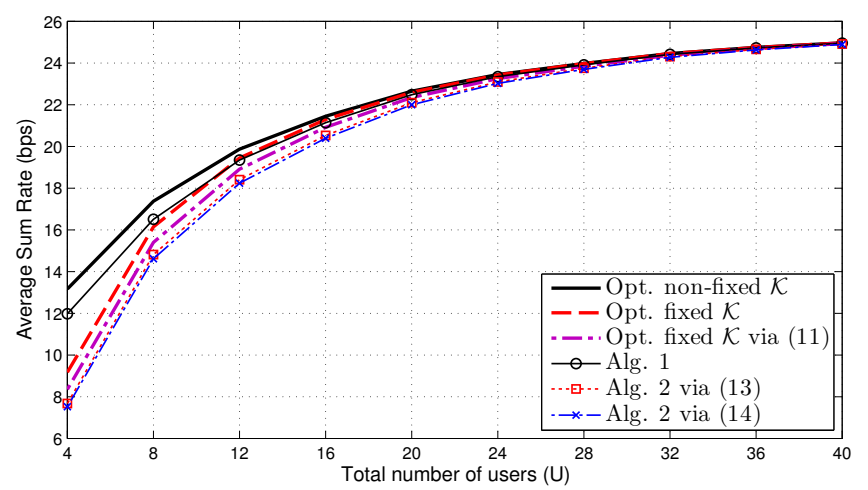

Fig. 1: Average sum rate (bps) versus user diversity $U$ for $N=4$.

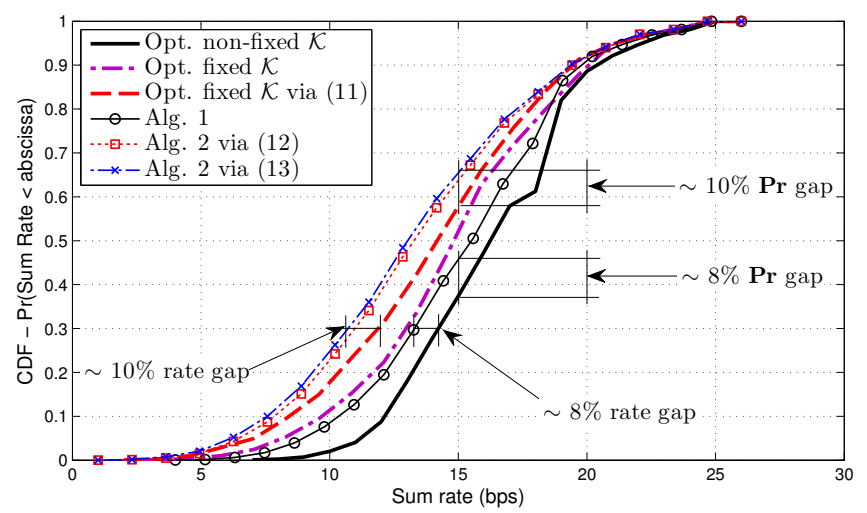

Fig. 2: Cumulative Distribution Function of the average sum rate for $U=8$.

\section{CONCLUSION}

In this paper we present two approaches to solve the problem of joint power and modulation allocation in interference limited systems using the Perron-Frobenius theory. The sum rate maximization problem is addressed by reaching feasibility via target relaxation and a subset of feasible links with their

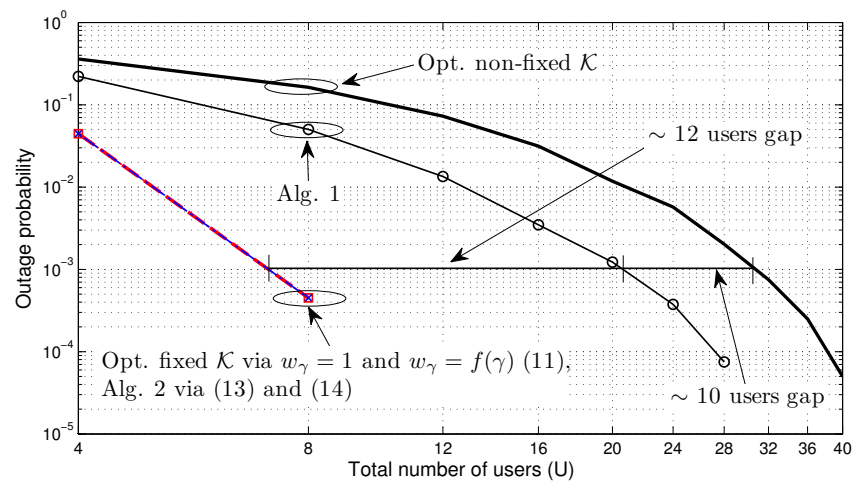

Fig. 3: Outage probability versus user diversity $U$.

respective targets and powers is found. Additionally, we solve the sum rate maximization problem when constraints on the subset of feasible links are imposed. We propose an algorithm for target tightening that exploits a criterion derived from the Perron-Frobenius theory and a less complex approach that profits from the information provided by the power consumption. Future work will be focused in decentralized algorithms, considering that the powers also take values from a finite set.

\section{REFERENCES}

[1] C. W. Tan, M. Chiang, and R. Srikant, "Fast Algorithms and Performance Bounds for Sum Rate Maximization in Wireless Networks," Networking, IEEE/ACM Trans. on, vol. PP, no. 99, p. 1, 2012.

[2] M. Chiang and J. Bell, "Balancing supply and demand of bandwidth in wireless cellular networks: utility maximization over powers and rates," in INFOCOM IEEE, vol. 4, march 2004, pp. 2800 - 2811.

[3] D. Cai, C. W. Tan, and S. Low, "Optimal max-min fairness rate control in wireless networks: Perron-frobenius characterization and algorithms," in INFOCOM, 2012 Proceedings IEEE, 2012, pp. 648-656.

[4] H. Boche and M. Schubert, "Perron-root minimization for interferencecoupled systems with adaptive receive strategies," Commun., IEEE Trans. on, vol. 57, no. 10, pp. 3173 -3164, october 2009.

[5] M. Schubert and H. Boche, QoS-based resource allocation and transceiver optimization. Foundation and Trends in Communications and Information Theory, 2006.

[6] S. Stanczak, M. Wiczanowski, and H. Boche, Fundamentals of resource allocation in wireless networks: theory and algorithms. Springer, 2009.

[7] H. Mahdavi-Doost, M. Ebrahimi, and A. Khandani, "Characterization of SINR region for interfering links with constrained power," Information Theory, IEEE Trans. on, vol. 56, no. 6, pp. 2816 -2828, june 2010.

[8] M. Chiang, P. Hande, T. Lan, and C. W. Tan, Power Control in Wireless Cellular Networks. Foundation and Trends in Networking, 2008.

[9] R. B. Bapat and T. E. S. Raghavan, Nonnegative Matrices and Applications. Cambridge University Press, 1997.

[10] J. E. Gentle, Matrix Algebra: Theory, Computations, and Applications in Statistics. Springer, 2007.

[11] M. Andersin, Z. Rosberg, and J. Zander, "Gradual removals in cellular PCS with constrained power control and noise," in Personal, Indoor and Mobile Radio Commun., vol. 1, sep 1995, pp. 56 -60 vol.1.

[12] A. Perez-Niera and M. Campalans, Cross-Layer Resource Allocation in Wireless Communications: Techniques and Models from PHY and MAC Layer Interaction. Academic Press, 2010.

[13] R. Jain, W. Hawe, and D. Chiu, "A Quantitative measure of fairness and discrimination for resource allocation in Shared Computer Systems," DEC-TR-301, Tech. Rep., 1984.

[14] E. Castañeda, R. Samano, and A. Gameiro, "Low Complexity Scheduling Algorithm for the Downlink of Distributed Antenna Systems," in Vehicular Technology Conference IEEE (VTC 2013-Spring), jun 2013.

[15] M.2135-1. Guidelines for evaluation of radio interface technologies for IMT-Advanced, ITU-R, 2009. 